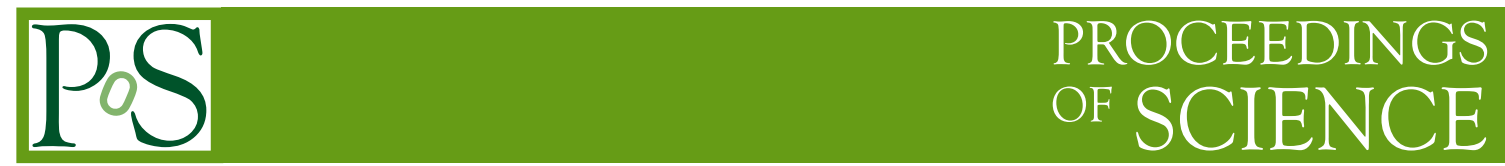

\title{
Cosmic Rays and the Interstellar Medium with the All-Sky Medium Energy Gamma-ray Observatory (AMEGO)
}

\section{Elena Orlando*t}

Kavli Institute for Particle Astrophysics and Cosmology and Hansen Experimental Physics Laboratory, Stanford University, CA (USA)

E-mail: orlandeledgmail.com

\section{Isabelle Grenier}

AIM, CEA, CNRS, Université Paris-Saclay, Université Paris Diderot, Sorbonne Paris Cite?, F-91191 Gif-sur-Yvette, France

\section{Vincent Tatischeff}

CSNSM, CNRS/Univ. Paris-Sud, Université Paris-Saclay, Orsay, France

\section{Andrey Bykov}

Ioffe Institute, St.Petersburg, Russia

\section{Andrew Strong}

Max Planck Institute for Extraterrestrial Physics, Garching, Germany

\section{Luigi Tibaldo}

IRAP, Université de Toulouse, CNRS, UPS, CNES, Toulouse, France

for the AMEGO Team $\ddagger$ 
We identify the scientific prospects for studying low-energy cosmic rays, the interstellar medium, and the associated gamma-ray emissions with a next-generation wide-field telescope from $200 \mathrm{keV}$ to $10 \mathrm{GeV}$. With improved angular resolution and more than an order of magnitude better sensitivity than previous instruments, the All-sky Medium Energy Gamma-ray Observatory (AMEGO) would allow for the first time to study in detail the low-energy cosmic rays, which play a fundamental role in the formation of stars and in the dynamics of the interstellar medium. It would allow mapping the cosmic-ray distribution in order to understand their propagation in the Galaxy. We discuss the importance of having such a telescope, and we present the predictions for the gamma-ray continuum both at large scale and in individual clouds, and for de-excitation nuclear lines.

This paper is based on the Astro2020: Decadal Survey on Astronomy and Astrophysics, science white papers, no. 151; Orlando E. et al. Bulletin of the American Astronomical Society, Vol. 51, Issue 3, id. 151 (2019) "Cosmic Rays and Interstellar Medium with Gamma-Ray Observations at MeV Energies" [1], where more details can be found.

36th International Cosmic Ray Conference -ICRC2019-

July 24th - August 1st, 2019

Madison, WI, U.S.A.

\footnotetext{
*Speaker.

${ }^{\dagger}$ E.O. acknowledges support from NASA Grants No. NNX16AF27G

${ }^{\ddagger}$ for collaboration list see PoS(ICRC2019)1177; https://asd.gsfc.nasa.gov/amego/team.html
} 


\section{Introduction}

Observations of the gamma-ray emission from the Milky Way from a few hundreds of $\mathrm{KeV}$ to a few tens of $\mathrm{GeV}$ provide insights on the Cosmic-Ray (CR) spectra, density, distribution, transport and interactions properties, and the CR interplay with the Interstellar Medium (ISM), even at large distances in the Galaxy. In fact, gamma-ray emission is mainly due to the interactions of Galactic CRs with gas and photons producing hadronic pion-decay emission, and producing leptonic inverse-Compton scattering and bremsstrahlung emission, as CRs propagate from their sources throughout the Galaxy. However, observations so far by INTEGRAL, Fermi LAT, and COMPTEL underline some discrepancies with present models, leaving open questions on the largescale distribution of CR sources, on CR transport mechanisms in the Galaxy, and on their density and spectral variation over the Galaxy (see e.g. [2, 3, 4] and reference therein). Moreover, lowenergy CRs are thought to be a fundamental component of the ISM, but their composition and flux are poorly known. In addition, the connection between low-energy CRs below a few GeV/nuc and galaxy evolution has started to be investigated only recently and is poorly understood (e.g. $[5,6,7,8,9,10,11])$.

The All-sky Medium Energy Gamma-ray Observatory (AMEGO) [12, 13, 14] is a probe class mission that will observe the entire sky in the energy range from $200 \mathrm{keV}$ to over $10 \mathrm{GeV}$, with more than an order of magnitude improvement in sensitivity relative to previous missions. This paper presents the scientific topics and expected outcomes that a mission from a few hundreds of $\mathrm{keV}$ to a few tens of $\mathrm{GeV}$ can target with the goal of understanding the role of CRs in the galaxies. A mission at $\mathrm{MeV}$ energy range would allow for the first time to study in detail the CRs with energies below a few GeV/nuc, which play a fundamental role in the formation of stars and on the dynamics in the Galaxy. CR sources, acceleration mechanisms, injection spectra into the ISM, transport properties, and their spectral and spatial distribution in the entire Galaxy, and in specific regions such as molecular clouds, star forming regions, and stellar clusters would be investigated for the first time for the entire energy band, and with unprecedented sensitivity and spatial resolution. As a consequence, distribution, acceleration, transport, and effects of CRs on the ISM and on the dynamics and evolution of the Galaxy can be finally understood.

\section{Scientific Topics and state of the art}

AMEGO will address three main scientific topics related to studies of CRs and ISM. They are described below.

\subsection{Large-scale interstellar emissions}

Observations at $\mathrm{MeV}$ energies would inform on the large-scale distribution of CR sources, on CR transport mechanisms in the Galaxy, including generation of winds, and on their density and spectral variation over the Galaxy (see e.g. [2]). The hadronic gas-related pion-decay emission is the major interstellar component at $\mathrm{GeV}$, while below $100 \mathrm{MeV}$ most of the emission comes from Inverse-Compton (IC) scattering and from bremsstrahlung emissions due to CR electrons $[15,16]$. AMEGO will for the first time allow to clearly separate emissions from CR nuclei and 
electrons. Moreover, it will also reveal the spatial and spectral distributions of the IC emission in the Galaxy [16], important for disentangling emission from unresolved sources (e.g. [17, 18]), the extragalactic diffuse gamma-ray background (e.g. [19]), or potential dark-matter signals (e.g. [20]). This also allows to infer the distribution of CR electrons, which better sample CR inhomogeneity, because they are affected by energy losses more strongly than nuclei, and they remain much closer to their sources. Moreover, observations of gamma rays below $100 \mathrm{MeV}$ by the same electrons that produce synchrotron emission in radio and microwaves provide firmer constraints on Galactic magnetic fields (see e.g. [21]). More details can be found in [1].

\subsection{ISM in dense regions}

$\mathrm{MeV}$ energies will allow resolving the $\mathrm{MeV}$ to $\mathrm{TeV}$ emission produced in and around a variety of star-forming regions, powered by stellar clusters of different masses and at different stages of evolution (see e.g. [3, 22]). The performance of AMEGO will open new avenues to (i) probe CRs in nearby clouds off the Galactic plane down to a half parsec scale above $1 \mathrm{GeV}$ and $10 \mathrm{pc}$ at 50 $\mathrm{MeV}$, (ii) compare the $\mathrm{CR}$ proton and electron spectra in and around nearby $\mathrm{OB}$ associations (e.g. in the Orion and Rosette nebulae), and (iii) to search for enhanced gamma-ray activity in massive $\mathrm{OB}$ associations beyond the few cases discovered at higher gamma-ray energies, such as the case of Cygnus X [23]. It will measure the energy distribution of the bulk of the CR nuclei, to estimate the CR pressure inside OB superbubbles [23], to follow the release and diffusion of CR electrons and nuclei around supernova remnants, and to measure how low-energy CRs get depleted inside dense clouds because of self-excited MHD turbulence [24]. AMEGO will also search for gammaray counterparts to synchrotron emitting protostars to set limits on CR production by jets which impacts the ionization, therefore the evolution of protostellar discs [25]. More details can be found in [1]

\subsection{De-excitation lines}

A very promising way to study the flux and composition of CR nuclei below the kinetic energy threshold for production of neutral pions ( $\sim 300 \mathrm{MeV}$ for $\mathrm{p}+\mathrm{p}$ collisions) would be to detect gamma-ray lines in the $0.1-10 \mathrm{MeV}$ range produced by nuclear collisions of CRs with interstellar matter [26]. Observations of this emission would be a clear proof of an important low-energy CR component in the Galaxy and it would allow to determine its composition, spectral and spatial distribution. The most intense lines are expected to be the same as those frequently observed from strong solar flares, i.e. lines from the de-excitation of the first nuclear levels in ${ }^{12} \mathrm{C},{ }^{16} \mathrm{O}$, ${ }^{20} \mathrm{Ne},{ }^{24} \mathrm{Mg},{ }^{28} \mathrm{Si}$, and ${ }^{56} \mathrm{Fe}$ [27]. The total nuclear line emission is also composed of broad lines produced by interaction of $\mathrm{CR}$ heavy ions with ambient $\mathrm{H}$ and $\mathrm{He}$, and of thousands of weaker lines. A particularly promising feature of the predicted gamma-ray spectrum is the characteristic bump in the range $3-10 \mathrm{MeV}$, which is produced by several strong lines of ${ }^{12} \mathrm{C}$ and ${ }^{16} \mathrm{O}$. Simulated gamma-ray line spectra of an individual nearby superbubble is reported in [28, 29]. The spectrum is dominated by both narrow and broad ${ }^{12} \mathrm{C}$ and ${ }^{16} \mathrm{O}$ lines providing a way to constrain low energy CR composition. More details can be found in [1]. 


\section{References}

[1] Orlando, E., Grenier, I., Tatischeff, V., et al. 2019, BAAS 51, 151

[2] Strong, A. W., Moskalenko, I. V., \& Ptuskin, V. S. 2007, Annual Review of Nuclear and Particle Science, 57, 285

[3] Grenier, I. A., Black, J. H., \& Strong, A. W. 2015, ARA\&A, 53, 199

[4] Drury, L. O. '., \& Strong, A. W. 2017, A\&A, 597, A117

[5] Enßlin, T. A., Pfrommer, C., Springel, V., \& Jubelgas, M. 2007, A\&A, 473, 41

[6] Persic, M., \& Rephaeli, Y. 2010, MNRAS, 403, 1569

[7] Padovani, M., Galli, D., \& Glassgold, A. E. 2009, A\&A, 501, 619

[8] Hanasz, M., Otmianowska-Mazur, K., Kowal, G., \& Lesch, H. 2009, A\&A, 498, 335

[9] Giacinti, G., Kachelrieß, M., \& Semikoz, D. V. 2012, Physical Review Letters, 108, 261101

[10] Recchia, S., Blasi, P., \& Morlino, G. 2016, MNRAS, 462, L88

[11] Pfrommer, C., Pakmor, R., Schaal, K., Simpson, C. M., \& Springel, V. 2017, MNRAS, 465, 4500

[12] McEnery, J. E. 2017, AAS/High Energy Astrophysics Division \#16, 103.13

[13] Caputo, R., Kislat, F., Racusin, J., et al. 2017, International Cosmic Ray Conference, 301, 783

[14] Moiseev, A., \& Amego Team 2017, International Cosmic Ray Conference, 301, 798

[15] Strong, A. W. 2011, Cosmic Rays for Particle and Astroparticle Physics, 473

[16] Orlando, E. 2018, MNRAS, 475, 2724

[17] Strong, A. W. 2007, AP\&SS, 309, 35

[18] Eckner, C., Hou, X., Serpico, P. D., et al. 2018, ApJ, 862, 79

[19] Ackermann, M., Ajello, M., Albert, A., et al. 2015, ApJ, 799, 86

[20] Ackermann, M., Ajello, M., Albert, A., et al. 2017, ApJ, 840, 43

[21] Orlando, E., \& Strong, A. 2013, MNRAS, 436, 2127

[22] Tibaldo, L., Digel, S. W., Casandjian, J. M., et al. 2015, ApJ, 807, 161

[23] Ackermann, M. et al. 2011, Science 334, 1103

[24] Dogiel, V. A., Chernyshov, D. O., Ivlev, A. V., et al. 2018, ApJ, 868, 114

[25] Padovani, M., Marcowith, A., Hennebelle, P., et al. 2016, A\&A, 590, A8

[26] Bykov, A. M. 2014, Astronomy Astrophysics Reviews 22, 77

[27] Ramaty, R., Kozlovsky, B., \& Lingenfelter, R. E. 1979, ApJS, 40, 487

[28] Tatischeff, V., \& Kiener, J. 2004, New Astronomy Reviews, 48, 99

[29] Benhabiles-Mezhoud, H., Kiener, J., Tatischeff, V., \& Strong, A. W. 2013, ApJ, 763, 98. Erratum: ApJ, 766, 139 